\title{
A physiological view and structures of mean residence times
}

\author{
Mária Ďurišová \\ Department of Pharmacology of Inflammation, Institute of Experimental Pharmacology and Toxicology, Slovak Academy \\ of Sciences, Dúbravská cesta 9, 84104 Bratislava, Slovak Republic
}

\begin{abstract}
The author's previous theoretical study described the determination of a physiologically realistic structure of a mean residence time of an intravenously administered drug (Ďurišová 2012). This study continues previous work and the aim is to determine physiologically realistic structures of mean residence times of a drug administered either orally $\left(M R T_{\mathrm{po}}\right)$ or intramuscularly $\left(M R T_{\mathrm{im}}\right)$. The determinations are based on the following assumption: a cardiopulmonary, portal, portal-venous, hepatic-portal subsystem, and a subsystem that mathematically represents non-eliminating tissues can be considered to be most important in terms of their impact on $M R T_{\mathrm{po}}$ and $M R T_{\mathrm{im}}$. If drug fate and disposition is a linear process, the used method allows developing mathematical models without any prior knowledge or hypothesis concerning drug fate and disposition. This is a great advantage of the method considered here when compared with compartment methods. The research presented in this study was aimed at contributing to the knowledge base of physiological origins of mean residence times.
\end{abstract}

Key words: Mean residence time - Pharmacokinetics - Circulatory model - Hepatic elimination

Abbreviations: ADME - absorption, distribution, metabolism, and elimination related dynamic system; MRT, mean residence time.

\section{Introduction}

A mean residence time $(M R T)$ is one of the major parameters that are used for investigations in pharmacokinetic studies. However, to the best of the author's knowledge, physiologically realistic structures of $M R T$ have not been published as yet, with the exception of the author's previous theoretical study (Ďrišová 2012). One explanation for this fact is probably that physiologically realistic structures of $M R T$ cannot be determined with tools commonly used in pharmacokinetics. Therefore, tools from the theory of dynamic systems, (see e.g., Gajic and Lelic 1996; Gajic and Lim 2001), were employed to determine a physiologically realistic structure of $M R T$ of a drug administered intravenously ( $\left.M R T_{\mathrm{iv}}\right)$ (Ďurišová 2012). This study describes the determinations of

Correspondence to: Mária Ďurišová, Department of Pharmacology of Inflammation, Institute of Experimental Pharmacology and Toxicology, Slovak Academy of Sciences, Dúbravská cesta 9, 84104 Bratislava, Slovak Republic

E-mail: Maria.Durisova@savba.sk physiologically realistic structures of $M R T$ of a drug administered either orally $\left(M R T_{\mathrm{po}}\right)$ or intramuscularly $\left(M R T_{\mathrm{im}}\right)$. The term ADME (absorption, distribution, metabolism, and elimination related dynamic system) was originally developed and used in the author's previous theoretical study. ADME related dynamic systems are abstract mathematical constructs, without any physiological relevance. ADME is a well-known acronym in pharmacokinetics (see e.g., Eddershaw et al. 2000). A design of an ADME related dynamic system is a simple procedure, it can be performed in the following way: a function approximating a drug input into the body is used as an input to an ADME related dynamic system, and a function approximating a response of the body to the drug input is used as an output of ADME related dynamic system (Ďurišová et al. 1995; Dedík and Ďurišová 1996; Ďurišová and Dedík 1997; Tvrdoňová et al. 2009). Thereafter, the ADME related dynamic systems are simply called "the dynamic systems".

It is well known that a dynamic process associated with drug fate and disposition is controlled by several dynamic mechanisms and influenced not only by diverse dynamic 
interactions between the drug administered and physiological environment but also by various factors (Chiou 1983; Verotta et al. 1991). Therefore, several studies described investigations of the dynamic process associated with drug fate and disposition with the aid of dynamic systems. The investigations were performed in the following way: (a) dynamic systems were defined, (b) optimal circulatory models of the dynamic systems were selected through the Akaike information criterion (Akaike 1974) and (c) parameters of the optimal models were estimated (Durišová et al. 1995; Ďurišová and Dedík 1997; Dedik et al. 2009).

The terminology used in the current theoretical study is commonly used in studies of dynamic systems. It is significantly different from the terminology commonly used in pharmacokinetic studies. The essential difference is that between the physiological nature of the information conveyed by a physiological system and the functional nature of the information conveyed by the dynamic systems used in the current theoretical study. In the current theoretical study, the dynamic systems are used as means to mathematically represent dynamic processes associated with drug fate and disposition (Weiss and Pang 1992; Xiao et al. 2012). Another difference is related to the use of the term „dynamic": In pharmacokinetics, the term "dynamic" is widely used in descriptions of drug actions (Zuideveld et al. 2002). In the current theoretical study the term "dynamic" is used to indicate continuous changes in processes associated with drug fate and disposition. The differences in terminology outlined above do not have any consequence on pharmacokinetics.

\section{Methods}

The physiologically realistic structures of $M R T_{\mathrm{po}}$ and $M R T_{\text {im }}$ are determined using two theoretical examples, two ADME related dynamic systems, and the same method as that previously described (Ďurišová 2012). The method considered here can be briefly described as follows: The dynamic process associated with drug fate and disposition was regarded as a result of repetitive passes of the drug around the blood circulation (Cutler 1979). The determination of a physiologically realistic structure of $M R T_{\mathrm{iv}}$ was performed in the following manner: In the first step, a theoretical example was created for illustrative purposes. In the second step a dynamic system was defined that mathematically represented the dynamic process associated with drug fate and disposition after the intravenous drug administration. In the third step, a circulatory model of the system defined was developed (Bassingthwaighte and Ackerman 1967; Weiss and Föester 1979). In the last step, a circulatory model developed and the method previously published (Ďurišová et al. 1995) were used to determine a physiologically realistic structure of $M R T_{\mathrm{iv}}$.
In this study, the determinations of the physiologically realistic structures of $M R T_{\mathrm{po}}$ and $M R T_{\mathrm{im}}$ are performed analogously to that previously described (Ďurišová 2012). The determinations consist of these steps: In the first step, the following assumptions were made: (a) drug fate and disposition was a linear dynamic process, (b) the drug was mainly eliminated by the biliary route (c) the drug was not bound to plasma proteins or tissues, (d) the drug was orally administered in a tablet which was rapidly dissolved in gastrointestinal fluid, (e) the dissolved drug was rapidly emptied via the bile into the small intestine and is then reabsorbed into the blood circulation, (f) no barriers to the distribution (or elimination) of the drug existed. In the second step, the dynamic systems $H_{\mathrm{po}}$ and $H_{\mathrm{im}}$ were defined, that mathematically represented the dynamic processes associated with drug fate and disposition after an oral and intramuscular administration, respectively. In the third step, circulatory models of the dynamic systems $H_{\mathrm{po}}$ and $H_{\mathrm{im}}$ were developed, and the transfer functions $H_{\mathrm{po}}(\mathrm{s})$ and $H_{\mathrm{im}}(\mathrm{s})$ of the systems $H_{\mathrm{po}}$ and $H_{\mathrm{im}}$ were determined. In the fourth step $M R T_{\mathrm{po}}$ and $M R T_{\mathrm{im}}$ were described by the following general equations

$$
\begin{aligned}
M R T_{p 0} & =\frac{\lim _{s \rightarrow 0} \frac{d H_{p o}(s)}{d s}}{\lim _{s \rightarrow 0} H_{p 0}(s)} \\
M R T_{i m} & =\frac{\lim _{s \rightarrow 0} \frac{d H_{i m}(s)}{d s}}{\lim _{s \rightarrow 0} H_{i m}(s)}
\end{aligned}
$$

in the Laplace (s) domain (Yamaoka et al. 1978; Ďurišová et al. 1995). In the last step the physiologically realistic structures of $M R T_{\mathrm{po}}$ and $M R T_{\mathrm{im}}$ are determined, using Eqs. (1), (2), the transfer functions $H_{\mathrm{po}}(\mathrm{s}), H_{\mathrm{im}}(\mathrm{s})$, the circulatory models developed, the method previously published (Ďurišová et al. 1995), and all assumptions made until now.

\section{Results}

The mean residence time of an intravenously administered drug

The physiologically realistic structure of $M R T_{\mathrm{iv}}$ determined is described by the following equation:

$$
M R T_{i v}=F_{c p}+F_{p}+F_{h}+F_{o}+F_{r} .
$$

As seen, the right-hand side of Eq. (3) contains five additive terms. The term $F_{\mathrm{cp}}$ describes the contribution to $M R T_{\mathrm{iv}}$ by the dynamic processes associated with the transport of the drug through the cardiopulmonary subsystem $H_{\text {cp. }}$. The term $F_{\mathrm{p}}$ describes the contribution to $M R T_{\mathrm{im}}$ by the dynamic 
process associated with the transport of the drug through the portal-venous subsystem $H_{\mathrm{p}}$. The term $F_{\mathrm{h}}$ describes the contribution to $M R T_{\mathrm{im}}$ by the dynamic process associated with the transport of the drug through the hepatic-portal subsystem $H_{\mathrm{h}}$. The term $F_{0}$ describes the contribution to $M R T_{\text {im }}$ by the dynamic processes associated with the transport of the drug through the subsystem $H_{0}$. The subsystem $H_{0}$ mathematically represents drug fate and disposition in non-eliminating tissues. If the drug is subject to the enterohepatic circulation (EHC) (Shepard et al. 1989; Tvrdoňová et al. 2009), the term $F_{\mathrm{r}}$ describes the contribution to $M R T_{\mathrm{im}}$ by the dynamic processes associated with the transport of the drug through the subsystem $H_{\mathrm{r}}$. The subsystem $H_{\mathrm{r}}$ mathematically represents the dynamic process associated with the EHC, and the terms $F_{\mathrm{p}}, F_{\mathrm{h}}$ and $F_{0}$ are as follows:

$$
\begin{aligned}
& F_{p}=\frac{Q_{h}-C l_{h}}{C l_{h}} \frac{Q_{p}}{Q_{h}} M T_{p} \\
& F_{h}=\frac{Q_{h}-C l_{h}}{C l_{h}} M T_{h} \\
& F_{o}=\frac{Q_{o}}{C l_{h}} M T_{o} .
\end{aligned}
$$

In Eqs. (4-6), $Q_{p}$ is blood flow in the portal vein, $Q_{0}$ is blood flow in non-eliminating tissues, $\mathrm{Cl}_{\mathrm{h}}$ is the hepatic clearance, $M T_{\mathrm{p}}$ is the mean transport time of the drug through the portal-venous subsystem, $M T_{\mathrm{h}}$ is the mean transport time of the drug through the hepatic-portal subsystem $H_{\mathrm{h}}, Q_{\mathrm{h}}$ is blood flow in the hepetic subsystem, and $M T_{0}$ is the mean transport time of the drug through the subsystem $H_{0}$ where

$$
M T_{o}=\frac{\sum_{i=1}^{q} Q_{i} \cdot M T_{i}}{Q_{o}}
$$

and finally $M T_{\mathrm{i}}$ is the mean transport time of the drug through non-eliminating tissues, the $i$ subscript specifies a tissue (Valentinutzzi 1971; Waterhouse and Keilson 1972).

The mean residence times of a drug administered either orally $\left(M R T_{p o}\right)$ or intramuscularly $\left(M R T_{i m}\right)$

The circulatory models developed are shown in Fig. 1 and Fig. 2. The physiologically realistic structure of the mean residence $M R T_{\text {po }}$ of an orally administered drug is described by the following equation

$$
M R T_{p o}=M T_{s}+M T_{p}+M T_{h}+M R T_{i v}
$$

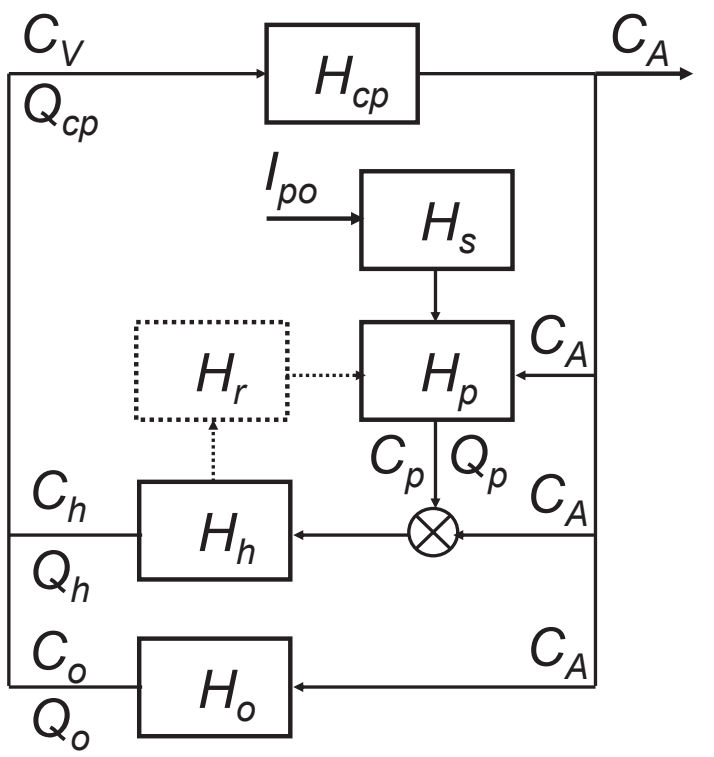

Figure 1. The circulatory model of the dynamic system $H_{\text {po }}$ which mathematically represents dynamic process of drug fate and disposition after an oral drug administration. $I_{\text {po }}$ is oral drug administration. $\mathrm{C}_{\mathrm{A}}$ is the concentration-time profile of the drug in arterial blood. $\mathrm{C}_{\mathrm{V}}$ is the concentration-time profile of the drug in venous blood. $H_{\mathrm{pc}}$ is the cardiopulmonary subsystem. $H_{\mathrm{p}}$ is the portal-venous subsystem. $H_{\mathrm{h}}$ is the hepatic subsystem. $H_{\mathrm{o}}$ is the subsystem representing non-eliminating tissues. $H_{\mathrm{r}}$ is the subsystem representing the enterohepatic cycling. $\mathrm{C}_{\mathrm{p}}$ is the concentration-time profile of the drug in the subsystem $H_{\mathrm{p}}$. $\mathrm{C}_{\mathrm{h}}$ is the concentration-time profile of the drug in the subsystem $H_{\mathrm{h}}$. $\mathrm{C}_{\mathrm{o}}$ is the concentrationtime profile of the drug in the subsystem $H_{\mathrm{o}}, \mathrm{O}_{\mathrm{cp}}, Q_{\mathrm{p}}, Q_{\mathrm{h}}, Q_{\mathrm{o}}$ are the blood flows through the subsystems specified by a subscript. The symbol $\otimes$ denotes a summation operator.

In Eq. (8), $M T_{\mathrm{s}}$ is the mean transport time of the drug through the subsystem $H_{\mathrm{s}}$. The subsystem $H_{\mathrm{s}}$ mathematically represents the following processes: disintegration of a tablet, the liberation of the drug from the tablet, drug dissolution, hepatic and intestinal first-pass effects (if present), and gastric emptying. $M T_{\mathrm{p}}$ is the mean transport time of the drug through the portal subsystem $H_{\mathrm{p}} . M T_{\mathrm{h}}$ is the mean transport time of the drug through the hepatic-portal subsystem $H_{\mathrm{h}} \cdot M R T_{\mathrm{iv}}$ in Eq. (8) is the same as that in Eq. (3). The sum of the mean transport times $M T_{\mathrm{s}}+M T_{\mathrm{p}}+M T_{\mathrm{h}}$ in Eq. (8) is mean transport time of the drug from the gastrointestinal tract to the blood circulation. If the drug is not subject to the EHC the term $F_{\mathrm{r}}$ in Eq. (3) is zero, and the term $F_{\mathrm{cp}}$ is as follows:

$$
F_{p o}=\frac{Q_{c p}}{C l} M T_{c p}
$$

If the drug is subject to the EHC, the term $F_{\mathrm{r}}$ in Eq. (3) is described by the following equation 


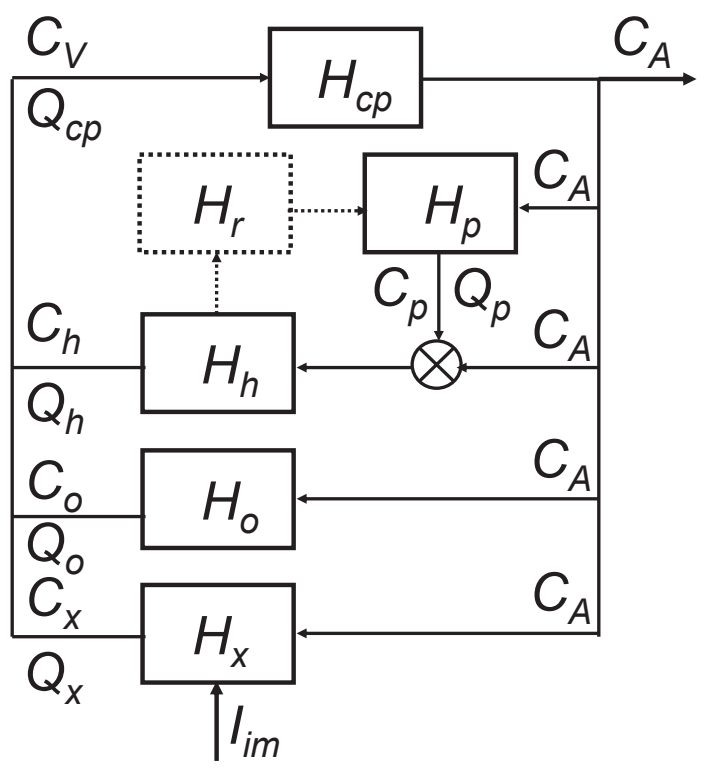

Figure 2. The circulatory model of the dynamic systems $H_{\mathrm{im}}$ which mathematically represents dynamic process of drug fate and disposition after an intramuscular administration. $\mathrm{I}_{\mathrm{im}}$ is the intramuscular drug administration. For the meaning of other symbols, see Figure 1.

$$
F_{r}=f_{r} \frac{Q_{h}-C l_{h}}{Q_{h}-f_{r} C l_{h}}\left(M T_{p}+M T_{h}+M T_{r}\right)
$$

In Eq. (10) $M T_{\mathrm{r}}$ is the mean transport time of the drug through the subsystem $H_{\mathrm{r}}$ and the coefficient $\mathrm{f}_{\mathrm{r}}$ where $0 \leq \mathrm{f}_{\mathrm{r}}$ $<1$ is the fraction of the drug that is subject to the EHC.

The physiologically realistic structure of the mean residence $M R T_{\text {im }}$ of an intramuscularly administered drug is described by the following equation

$$
M R T_{i m}=M T_{x}+M R T_{i v}
$$

where $M T_{\mathrm{X}}$ is the mean transport time of the drug through the subsystem $H_{\mathrm{x}}$ i.e. the mean time of the transport drug from the injection site to the blood circulation. $M R T_{\mathrm{iv}}$ in Eq. (11) is the same as that in Eqs. (3) and (8).

\section{Discussion}

The current theoretical study is based on concepts borrowed from the theory of dynamic systems. Being a researcher in pharmacokinetics and using tools from the theory of dynamic systems is almost like begging for trouble. A quick look through handbooks on the theory of dynamic systems (see e.g. the books cited above) reveals an amassment of abstract terms and mathematical equations most of which are simply not accessible for a mathematically untrained reader, which a researcher in pharmacokinetics is likely to be. Therefore a prominent feature of the current theoretical study is its non-technical style which makes it easily accessible to a reader without any knowledge of the theory of dynamic systems. Moreover, the physiologically realistic structures determined are fairly simple, and contain only blood flows, MRTs, and clearances related to individual body parts. They do not require a sophisticated knowledge of the theory of dynamic systems understand them.

The theory of dynamic systems incorporates computational and experimental approaches, and provides a number of new visions, strategies, and practices for engineering in pharmacokinetics. This theory offers a framework for using general strategies to construct mathematical models of a wide variety of dynamic processes in pharmacokinetics such as dissolution, gastric emptying, absorption, etc.

Transfer functions are key tools to investigate dynamic systems in the theory of dynamic systems. A mathematical idea to describe drug fate and disposition with a transfer function is not recent. A transfer function was introduced to pharmacokinetics by Professor Giorgio Segré (Segré 1988).

Such a complicated dynamic process as drug fate and disposition cannot be exactly described by a mathematical model, and always there are inaccuracies in a model due to un-modeled characteristics of the modeled process. This is also the case for the circulatory models developed in the current theoretical study. In spite of this, using the circulatory models developed the physiologically realistic structures of $M R T_{\mathrm{po}}$ and $M R T_{\mathrm{im}}$ are successfully determined.

The circulatory model of the dynamic system $H_{\mathrm{po}}$ and the physiologically realistic structure of $M R T_{\text {po }}$ reflect the fact that an orally administered drug has to pass through the gastrointestinal tract and liver before entering the blood circulation (Chiou 1983). This drug transport is mathematically represented by the subsystems $H_{\mathrm{p}}, H_{\mathrm{h}}$ and $H_{\mathrm{r}}$ in the circulatory model of the system $H_{\mathrm{po}}$. It follows then that, the circulatory model developed correctly approximates main processes in the body after an oral drug administration. In the circulatory model of the system $H_{\text {po }}$ shown in Figure 2, the subsystem $H_{\mathrm{r}}$ is illustrated by dotted lines, because the EHC may be present or not present.

The MRTs, determined by conventional pharmacokinetic methods are single-number summaries for characteristics of responses of the body to drug inputs. In contrast the physiologically realistic structures of $M R T_{\text {po }}$ and $M R T_{\text {im }}$ may provide novel information not apparent from $M R T_{\text {po }}$ and $M R T_{\mathrm{im}}$ determined by conventional pharmacokinetic methods. In this way the physiologically realistic structures of $M R T_{\text {po }}$ and $M R T_{\mathrm{im}}$ may expand the understanding of $M R T_{\text {po }}$ and $M R T_{\mathrm{im}}$. 
As can be seen in an overwhelming number of studies, $M R T s$, in spite of their approximate nature, have become valuable tools in pharmacokinetics (Cheng and Jusko 1989; Plusquellec and Houin 1990; Verotta et al. 2001; García-Meseguer et al. 2003). Taking into account this fact, the goal of the current theoretical study was to introduce the physiologically realistic structures of MRTs to pharmacokinetics.

The current theoretical tries to arouse interest of researchers and practitioners in pharmacokinetics and in physiology in the theory of dynamic systems. Differences between traditional pharmacokinetic approaches and approaches based on the theory of dynamical systems to MRTs are left unexplained. Instead of a comparison between approaches, the current theoretical study gives rise to a reasonable expectation that the use of the structures determined for the enhancing understanding of MRTs is not a utopian goal. The physiologically realistic structures determined have not been experimentally validated up to now. Their validity can be verified by further investigations, mainly experimental investigations. For this reason a full pharmacokinetic exploitation of the structures of $M R T_{\mathrm{po}}$ and $M R T_{\mathrm{im}}$ lies far in the future.

In this study an attempt is made to contribute to the theoretical base of pharmacokinetics, without an intention to criticize traditional approaches to $M R T_{\mathrm{po}}$ and $M R T_{\mathrm{im}}$.

Acknowledgements. The research work of the author in the 7FP-Project "EU-Network of Excellence, Virtual Physiological Human" led to the preparation of the current theoretical study. The author gratefully acknowledges the financial support obtained from the Slovak Academy of Sciences during the preparation of the current theoretical study. Last, but not least, the author is indebted to Ladislav Dedík for his advice on the determination of the physiologically realistic structures in the current theoretical study.

\section{References}

Akaike H. (1974): A new look at the statistical model identification. Automatic Control, IEEE Transactions on 19, 716-723

Bassingthwaighte J. B., Ackerman F. H. (1967): Mathematical linearity of circulatory transport. J. Appl. Physiol. 22, 879-888

Cutler D. J. (1979): A recirculation model for drug disposition. J. Pharmacokinet. Biopharm. 7 101-116 http://dx.doi.org/10.1007/BF01059445

Cheng H., Jusko W. J. (1989): Application of mean residence-time concepts to pharmacokinetic systems with noninstantaneous input and nonlinear elimination. Pharm. Res. 6, 4-12 http://dx.doi.org/10.1023/A:1015883131875

Chiou W. L. (1983): Mean hepatic transit time in the determination of mean absorption time. J. Pharm. Sci. 72 1365-1368 http://dx.doi.org/10.1002

Dedík L., Ďurišová M. (1996): CXT-MAIN: A software package for determination of the analytical form of the pharmacoki- netic system weighting function. Comput. Methods Programs Biomed. 51, 183-192 http://dx.doi.org/10.1016/S0169-2607(96)01770-1

Dedík L., Tvrdoňová M., Ďrišová M., Penesová A., Miklovičová D., Kozlovský M. (2009): Computer controlled sequential simulation method: reconsidering evaluation of measurements from frequently sampled intravenous glucose tolerance test. Comput. Methods Programs Biomed. 95, 1-9 http://dx.doi.org/10.1016/j.cmpb.2009.01.001

Ďurišová M., Dedík L., Balan M. (1995): Building a structured model of a complex pharmacokinetic system with time delays. Bull. Math. Biol. 57, 787-808 http://dx.doi.org/10.1007/BF02458295

Ďurišová M., Dedík L. (1997): Modeling in frequency domain used for assessment of in vivo dissolution profile. Pharm. Res. 14, 860-864 http://dx.doi.org/10.1023/A:1012139530965

Durišová M. (2012): Physiologically based structure of mean residence time. Sci. World J. 2012, 610631

Eddershaw L. J., Beresford A. P., Bayliss M. K. (2000): ADME/PK as a part of a rational approach to drug discovery. Drug Discov. Today 5, 409-414 http://dx.doi.org/10.1016/S1359-6446(00)01540-3

Gajič Z., Lelič M. (1996): Modern Control Systems, Engineering. Prentice-Hall International Series in Systems and Control Engineering London

Lim M. (2001): Optimal Control of Singularly Perturbed Linear Systems and Applications. Marcel Dekker, Inc., New York

García-Meseguer M. J., Vidal de Lambra J. A., García-Moreno M., García-Cánovas F., Havsteen B. H., Varón R. (2003): Mean residence times in linear compartmental systems. Symbolic formulae for their direct evaluation. Bull. Math. Biol. 65, 279-308 http://dx.doi.org/10.1016/S0092-8240(02)00096-4

Plusquellec Y., Houin G. (1992): Compartmental mean residence time in a mammillary model with an effect compartment after intravenous or oral administration. J. Biomed. Eng. 14, 521-526 http://dx.doi.org/10.1016/0141-5425(92)90107-V

Segre G. (1988): The sojourn time and its prospective use in pharmacology. J. Pharmacokinet. Biopharm. 7, 657-666

Shepard T. A., Lockwood G. F., Aarons L. J., Abrahams I. D. (1989): Mean residence time for drugs subject to enterohepatic cycling. J. Pharmacokinet. Biopharm. 17, 327-345 http://dx.doi.org/10.1007/BF01061900

Tvrdoňová M., Chrenová J., Rausová Z., Miklovičová D., Ďurišová M., Mircioiu C., Dedík L. (2009): Novel approach to bioequivalence assessment based on physiologically realistic model. Int. J. Pharm. 380, 89-95 http://dx.doi.org/10.1016/j.ijpharm.2009.07.004

Valentinutzzi M. E. (1971): A mathematical model of the hepatic portal system. Med. Biol. Eng. 3, 213-220 http://dx.doi.org/10.1007/BF02474816

Verotta D., Sheiner L. B., Beal L. S. (1991): Mean time parameters for generalized physiological flow models (semihomogeneous linear systems). J. Pharmacokinet. Biopharm. 19, 319-331 http://dx.doi.org/10.1007/BF03036254

Waterhouse C., Keilson J. (1972): Transfer times across the human body. Bull. Math. Biophys. 34, 33-44 http://dx.doi.org/10.1007/BF02477022 
Weiss M., Föester W. (1979): Pharmacokinetic model based on circulatory transport. Eur. J. Clin. Pharmacol. 4, 287-293 http://dx.doi.org/10.1007/BF00608408

Weiss M., Pang K. S. (1992): Dynamics of drug distribution. I. Role of the second and third curve moment. J. Pharmacokinet. Biopharm. 20, 253-278 http://dx.doi.org/10.1007/BF01062527

Xiao H., Song H., Yang Q., Cai H., Qi R., Yan L., Liu S., Zheng Y., Huang T., Liu T., Jing X. (2012): A prodrug strategy to deliver cisplatin (IV) and paclitaxel in nanomicelles to improve efficacy and tolerance. Biomaterials 33, 6507-6519

http://dx.doi.org/10.1016/j.biomaterials.2012.05.049
Yamaoka K., Nakagawa T., Uno T. (1978): Statistical moments in pharmacokinetics. J. Pharmacokinet. Biopharm. 6, 547-558 http://dx.doi.org/10.1007/BF01062109

Zuideveld K. P., van Gestel A., Peletier L. A., Van der Graaf P. H., Danhof M. (2002): Pharmacokinetic - pharmacodynamic modelling of the hypothermic and corticosterone effects of the 5-HT1A receptor agonist flesinoxan. Eur. J. Pharmacol. 445, 43-54 http://dx.doi.org/10.1016/S0014-2999(02)01665-5

Received: April 29, 2013

Final version accepted: July 15, 2013 